\title{
Association of Back Pain with All-Cause and Cause-Specific Mortality Among Older Women: a Cohort Study
}

\author{
Eric J. Roseen, DC MSc' ${ }^{7}$, Michael P. LaValley, PhD², Shanshan Li, ScD ${ }^{3}$, Robert B. Saper, MD MPH', \\ David T. Felson, MD MPH', and Lisa Fredman, $\mathrm{PhD}^{4}$ For the Study of Osteoporotic Fractures
}

'Department of Family Medicine, Boston Medical Center, One Boston Medical Center Place, Boston, MA, USA; ${ }^{2}$ Department of Biostatistics, Boston University School of Public Health, Boston, MA, USA; ${ }^{3}$ Clinical Epidemiology Research and Training Unit, Boston University School of Medicine, Boston, MA, USA; ${ }^{4}$ Department of Epidemiology, Boston University School of Public Health, Boston, MA, USA.

BACKGROUND: The impact of back pain on disability in older women is well-understood, but the influence of back pain on mortality is unclear.

OBJECTIVE: To examine whether back pain was associated with all-cause and cause-specific mortality in older women and mediation of this association by disability.

DESIGN: Prospective cohort study.

SETTING: The Study of Osteoporotic Fractures.

PARTICIPANTS: Women aged 65 or older.

MEASUREMENT: Our primary outcome, time to death, was assessed using all-cause and cause-specific adjusted Cox models. We used a four-category back pain exposure (no back pain, non-persistent, infrequent persistent, or frequent persistent back pain) that combined back pain frequency and persistence across baseline (1986-1988) and first follow-up (1989-1990) interviews. Disability measures (limitations of instrumental activities of daily living [IADL], slow chair stand time, and slow walking speed) from 1991 were considered a priori potential mediators.

RESULTS: Of 8321 women (mean age $71.5, \mathrm{SD}=5.1$ ), 4975 (56\%) died over a median follow-up of 14.1 years. A higher proportion of women with frequent persistent back pain died (65.8\%) than those with no back pain (53.5\%). In the fully adjusted model, women with frequent persistent back pain had higher hazard of all-cause (hazard ratio $[\mathrm{HR}]=1.24$ [95\% CI, 1.11-1.39]), cardiovascular $(\mathrm{HR}=1.34$ [CI, 1.12-1.62] $)$, and cancer $(\mathrm{HR}=1.33$, [CI 1.03-1.71]) mortality. No association with mortality was observed for other back pain categories. In mediation analyses, IADL limitations explained $47 \%$ of the effect of persistent frequent back pain on all-cause mortality, slow chair stand time, and walking speed, explained $27 \%$ and $24 \%$ (all significant, $p<0.001$ ), respectively.

LIMITATIONS: Only white women were included.

$\overline{\text { Prior Presentations Part of this work was presented as a poster at the }}$ International Back and Neck Pain Forum (September 2017) in Oslo, Norway. Additionally, we presented our main findings in an oral presentation at the Society for Epidemiology Research meeting (June 2018) in Baltimore, $M D$

Electronic supplementary material The online version of this article (https://doi.org/10.1007/s11606-018-4680-7) contains supplementary material, which is available to authorized users.

Received April 6, 2018

Revised July 6, 2018

Accepted September 13, 2018

Published online October 22, 2018
CONCLUSION: Frequent persistent back pain was associated with increased mortality in older women. Much of this association was mediated by disability.

KEY WORDS: back pain; mortality; disability; mediation.

J Gen Intern Med 34(1):90-7

DOI: $10.1007 / \mathrm{s} 11606-018-4680-7$

(c) Society of General Internal Medicine 2018

\section{INTRODUCTION}

Back pain is the leading cause of disability worldwide. ${ }^{1}$ Women aged 40-80 years have the highest prevalence of back pain. $^{2}$ Additionally, women report more frequent and disabling back pain compared to men. ${ }^{3-5}$ While the relationship between back pain and disability in older women is wellestablished, ${ }^{6,7}$ back pain's impact on mortality remains unclear.

Nine studies evaluating the relationship between back pain and mortality have produced conflicting results. ${ }^{8-16}$ Two studies separated back pain into multiple phenotypes based on pain frequency or impact on physical function, both found an elevated risk of mortality among older women. ${ }^{14,15}$ Other studies dichotomized back pain exposure; of these, two observed a small increase in mortality among older adults but did not provide sex-specific results, ${ }^{13}, 16$ and five found no association. $^{8-12}$ All previous studies measured back pain at a single time point. However, the impact of back pain on disability or general health varies substantially, and infrequent or temporary symptoms may not have major health consequences. Categorizing frequency and persistence of back pain symptoms over time may provide better understanding of these relationships. Thus, there is a need to elucidate whether back pain is associated with mortality in older adults, and older women may represent a high-risk group.

Back pain may increase mortality risk through several mechanisms. Disability might be one mechanism: studies found that disabling back pain increased mortality risk $^{16}$ and that baseline-level disability attenuated the association between back pain and mortality. ${ }^{13}$ However, previous studies have not examined whether consequences of back pain contributed to early death (i.e., mediators [e.g., disability]). Such 
mediation analyses could identify pathways by which back pain may increase the risk of mortality.

We assessed the impact of back pain on all-cause and causespecific mortality over 16 years in the Study of Osteoporotic Fractures (SOF), a large prospective cohort study of older community-dwelling women. In contrast to previous studies, we measured back pain frequency at two time points, thereby differentiating levels of back pain by frequency and persistence. Additionally, we assessed whether the association between back pain and mortality operated through subsequent disability ${ }^{17}$ (i.e., self-reported limitations in instrumental activities of daily living (IADLs) or performance-based physical function impairments [slow chair stand or walking speed]) and quantified the amount of these effects. We hypothesized that (1) compared to older women without back pain, those with frequent back pain persisting over 2 years would have the highest mortality risk and those with less frequent pain would have an intermediate risk of mortality over a 16 -year period; and (2) the association of back pain with mortality would be partially mediated by disability.

\section{METHODS}

\section{Study Design and Data Source}

SOF is a prospective cohort study of 9704 community-dwelling white women 65 years or older. SOF was originally designed to assess risk factors for the development of osteoporosis and osteoporotic fractures in healthy white women, as described elsewhere. ${ }^{18}$ Briefly, participants were enrolled from 1986 to 1988 from four US-based geographic regions (Baltimore, MD; Minneapolis, MN; Monongahela Valley, PA; Portland, OR). Non-white women and women unable to walk independently or who had bilateral hip replacements were excluded. Data were collected during clinical interviews and physical examinations at baseline and approximately every 2 years through 2008 .

\section{Study Cohort}

Our sample was comprised of SOF participants with selfreport information on back pain at baseline (visit 1 [V1], 1986-1988) and the first follow-up visit (visit 2 [V2], 19891990) who had complete information for covariables used in multivariable models. Variables for mediation analyses came from visit 3 (V3, 1991); therefore, participants who died before visit 3 were excluded from the mediation analyses. This study was approved by the Institutional Review Boards at each SOF clinical site and at Boston University Medical Campus.

\section{Assessment of Back Pain}

We created a four-category back pain variable: (1) no back pain, (2) non-persistent, (3) infrequent persistent, or (4) frequent persistent back pain. At V1 and V2, participants were asked, "Have you experienced back pain in the past 12 months?" and, if yes, "How often do you experience back pain?" Participants reporting back pain symptoms "most of the time" or "constantly" at both time points were classified as having "frequent persistent" back pain. Participants with back pain on both visits who reported back pain "some of the time" or "infrequently" at one or both visits were classified as having "infrequent persistent" back pain. Back pain reported at only one time point was characterized as "non-persistent" back pain. Participants who reported no back pain at both visits were classified as "no back pain." To assure this four-category classification was consistent with other SOF back pain measures, we compared it with other self-report measures: back pain intensity (severe, moderate, mild), back pain site(s) (lower, middle, upper), number of back pain sites (one, two, or three regions), back pain interference with activities of daily living (yes/no), and difficulty lifting 10 pounds due to back pain (none, some, much, unable). Additionally, reported age of first back pain episode ever was compared among exposure groups.

\section{Ascertainment of Deaths}

All-cause and disease-specific mortality was determined from V2 to visit 9 (V9, 2006-2008). In addition to comprehensive clinical visits approximately every 2 years, participants were contacted by mail or telephone every 4 months to assess outcomes, including vital status which was confirmed by review of death certificates. We used ICD-9 codes to classify deaths as due to cardiovascular disease $(401-404,410-414,425,428$, $429.2,430-438,440-444,798)$, cancer (140-239.9), and pulmonary $(415-417,480-487,490-496,500-508.8,510$ 519) diseases. ${ }^{19}$

\section{Potential Confounders}

Measurements of potential confounders were obtained from SOF V2 when available, since this was the most recent assessment of back pain; otherwise, they were obtained from V1. Sociodemographic variables included age, highest education level (high school graduate or less), currently married (yes/no), living alone (yes/no), and SOF site. Participants reported self-rated health (excellent vs. good/fair/poor) and smoking status (current/previous vs. never). Body mass index (BMI, calculated as weight in kilograms divided by height in meters squared) was measured and categorized as obese (BMI $>30.0 \mathrm{~kg} / \mathrm{m}^{2}$ ) or non-obese. Hypertension was defined as a systolic blood pressure above $140 \mathrm{mmHg}$ and/or diastolic blood pressure above $90 \mathrm{mmHg}$. Prevalent vertebral fractures (yes/no) were identified at V1 using vertebral morphometry. ${ }^{19}$ Participants self-reported ever being diagnosed (yes/no) with arthritis, hip pain, diabetes, stroke, breast cancer, and breast surgery. Additionally, participants reported falls, hospitalizations, or anxiety medication use in the previous year.

\section{Potential Mediators}

Three measures of disability from $\mathrm{V} 3$ were considered a priori potential mediators: IADL limitations, slow chair stand time, 
and slow walking speed. Participants were characterized as having "IADL limitations" if they self-reported difficulty with performing one or more of five IADL tasks: (1) walking 2-3 blocks, (2) climbing up 10 steps, (3) preparing a meal, (4) performing heavy housework, and (5) shopping for groceries or clothes. Slow chair stand time ${ }^{20}$ was based on taking longer than $17 \mathrm{~s}$ to stand from a seated position five times without using one's arms, using arms for assistance, or being unable to perform this task; otherwise, participants were classified as having "normal" performance. ${ }^{21,} 22$ Walking speed was measured as the average time of two trials of walking a 6-m course at usual pace. Slow walking speed was defined as slower than 1.0 m per second. ${ }^{21,22}$

\section{Statistical Analysis}

Kaplan-Meier survival curves were used to plot survival according to back pain group. Participants contributed person-time starting at V2 until death, withdrawal from SOF, or censoring at $\mathrm{V} 9 .{ }^{23}$ We used Cox proportional hazards regression to calculate hazard ratios (HR) and $95 \%$ confidence intervals (CI) of the association between each of the three back pain categories vs. no back pain with all-cause and causespecific mortality. Three multivariable models were conducted: age-adjusted, sociodemographic-adjusted, and fully adjusted including all potential confounders. Sensitivity analyses assessed the hazard of mortality associated with other self-reported back pain measures, described above.

We applied mediation methods ${ }^{24,25}$ to calculate the direct and indirect effects of back pain on all-cause mortality and the proportion of effect mediated by each measure of disability, adjusted for potential confounders (Appendix Fig. 1). The natural indirect effect (NIE) describes the extent that the association of back pain with mortality is attributable to each measure of disability; the residual natural direct effect (NDE) quantified the effect not mediated by these disability measures. Since these analyses require a dichotomous exposure, we compared "frequent persistent back pain" to the three other groups combined. Natural direct and indirect effects were calculated using logistic regression and presented as odds ratios (OR) and 95\% CI. The proportion of the total effect explained by the presence of each individual potential mediator (i.e., the proportion mediated on a risk difference scale) was calculated using the formula:

proportion mediated $=\left[\mathrm{OR}_{\mathrm{NDE}} \times\left(\mathrm{OR}_{\mathrm{NIE}}-1\right)\right] /\left[\left(\mathrm{OR}_{\mathrm{NDE}} \times \mathrm{OR}_{\mathrm{NIE}}\right)-1\right] \times 100$

SAS version 9.4 (SAS Institute, Cary, North Carolina) was used for all statistical analyses.

\section{RESULTS}

Of 9704 original SOF participants, 8915 had complete back pain data at V2; of these, 8321 had data on all covariates used in complete case analysis (Fig. 1). All were white women with a mean age of 71.5 years (SD 5.1) (Table 1). Approximately three-fourths of participants reported back pain and 9.5\% reported frequent persistent back pain. Participants with frequent persistent back pain were less likely to report excellent health and more likely to have co-morbid musculoskeletal conditions (e.g., prevalent vertebral fracture, arthritis, hip pain), but did not differ on age or marital status. Compared to the women included in this sample, the 789 SOF participants excluded had more prevalent vertebral fractures and reported worse self-rated health, but otherwise did not differ on sociodemographic or health characteristics. Baseline characteristics of participants in the analytic sample were similar to those included in the survival and mediation analyses (Appendix Table 1).

A total of 4590 participants $(55.2 \%)$ died over a median follow-up length of 14.1 years. A higher proportion of participants with frequent persistent back pain died (65.0\%) compared with those who reported no pain (52.9\%), non-persistent pain $(54.4 \%)$, or infrequent persistent back pain $(54.7 \%)$. Participants with frequent persistent back pain were more likely than others to report back pain of severe intensity, restrict activity due to back pain, and be unable to lift 10 pounds (Appendix Table 2). Moreover, participants with frequent persistent back pain reported an earlier mean age of onset than those with infrequent persistent back pain (45 vs. 53 years) or non-persistent back pain (61 years). Our fourcategory back pain measure was significantly correlated with other SOF back pain measures, with correlation coefficients ranging from 0.38 to 0.85 (Appendix Table 3).

Kaplan Meier curves showed significant differences in survival for all-cause, cardiovascular, cancer, and pulmonary deaths (Fig. 2, Appendix Fig. 2a-c). Compared with women without back pain, those with frequent persistent back pain had a $46 \%$ increase in the hazard of all-cause mortality in ageadjusted analyses ( $\mathrm{HR}=1.46,95 \%$ CI $1.32-1.61)$ (Table 2). This association was attenuated when adjusting for potential confounders (fully adjusted HR $=1.24,95 \%$ CI 1.11-1.39). Women with frequent persistent back pain also had an elevated hazard of cardiovascular, cancer, and pulmonary mortality (fully adjusted $\mathrm{HR}=1.34,95 \%$ CI $1.12-1.61$ and $1.33,95 \%$ CI $1.03-1.71$ and $1.32,95 \% 0.95-1.83$, respectively). We observed no increased hazard of all-cause or cause-specific mortality for non-persistent and infrequent persistent back pain.

Sensitivity analyses using other self-report measures of back pain yielded similar results to our primary analyses (Appendix Table 4). For example, while women who reported much difficulty lifting a 10-pound object due to back pain (vs. no difficulty) had a moderately increased hazard of all-cause mortality (fully adjusted HR $=1.57,95 \%$ CI $1.34-1.82$ ), those who reported any/unspecified back pain did not (fully adjusted $\mathrm{HR}=1.00,95 \%$ CI $0.94-1.07$ ).

In our analyses of mediation effects, having 1 or more IADL impairment mediated the association of frequent persistent back pain with all-cause mortality (fully adjusted indirect 


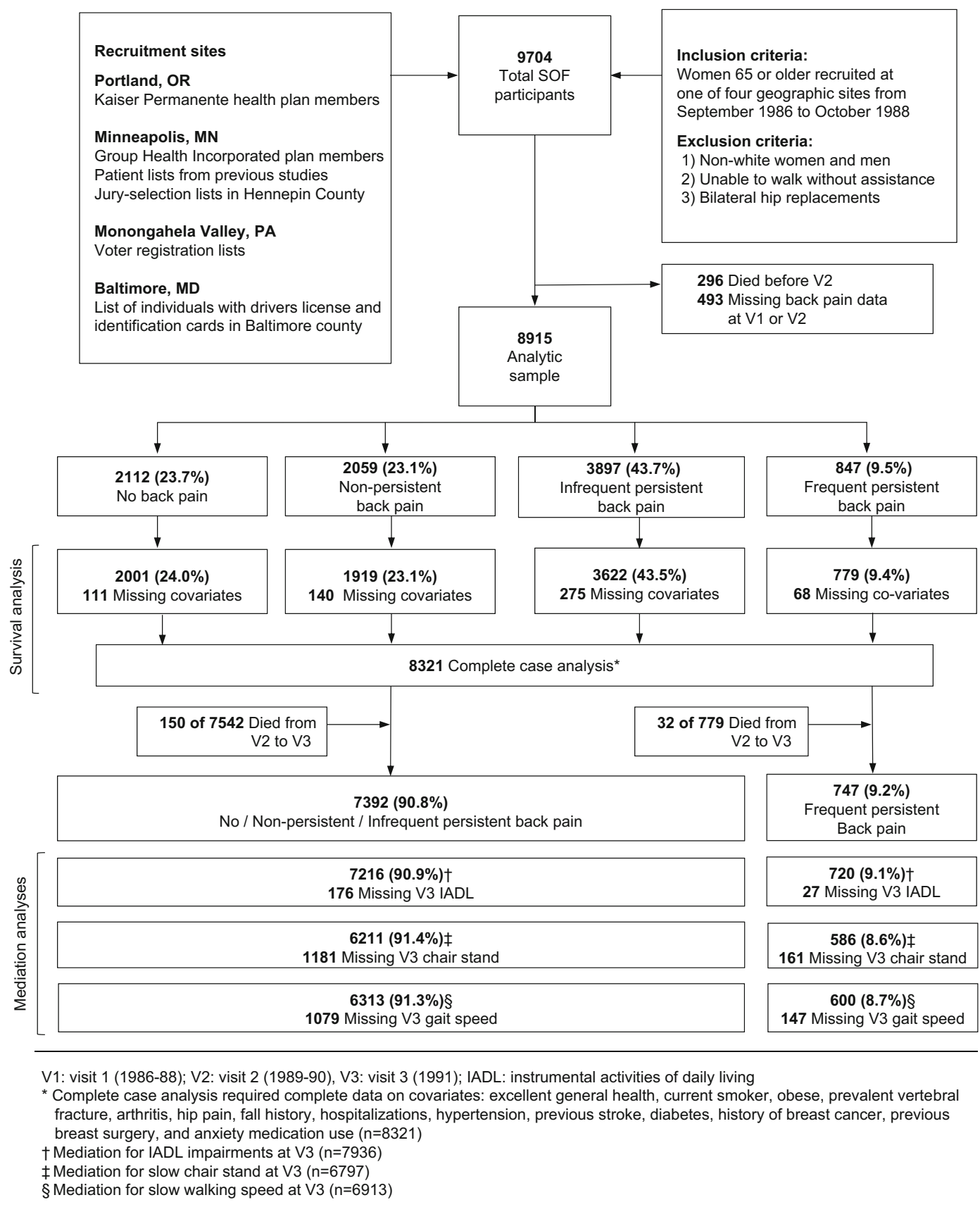

Fig. 1 Study flow diagram.

effect, $\mathrm{OR}=1.12,95 \% \mathrm{CI} 1.08-1.15)$, as did poor performance on chair stand (fully adjusted $\mathrm{OR}=1.05,95 \% \mathrm{CI}$ 1.03-1.08) and walking speed (fully adjusted $\mathrm{OR}=1.05$, 95\% CI 1.02-1.07) testing (Table 3). Similarly, while nearly half $(47 \%)$ of the mortality risk was mediated by IADL limitations, only a fourth of the overall effect was mediated by chair stand and walking speed (27\% and 24\%, respectively).

\section{DISCUSSION}

Among community-dwelling older white women followed for a median of 14.1 years, those reporting frequent persistent back pain had a $24 \%$ increased risk of all-cause mortality compared to participants without back pain. Frequent persistent back pain was also associated with elevated cardiovascular and cancer mortality. Participants with non-persistent or infrequent persistent back pain did not have a higher all-cause or disease-specific mortality. Limited ability to perform 1 or more IADLs was an important mediator, explaining half of this association, while slow chair stand and gait speed mediated one-fourth. These findings support our hypothesis that more frequent back pain persisting over 2 years is associated with subsequent mortality, and that this association is in part mediated by disability. 
Table 1 Baseline Characteristics of 8321 Older Women in the Study of Osteoporotic Fractures by Back Pain Exposure Status

\begin{tabular}{|c|c|c|c|c|}
\hline & $\begin{array}{l}\text { No } \\
\text { back pain }\end{array}$ & $\begin{array}{l}\text { Non-persistent } \\
\text { back pain }\end{array}$ & $\begin{array}{l}\text { Infrequent persistent } \\
\text { back pain }\end{array}$ & $\begin{array}{l}\text { Frequent persistent } \\
\text { back pain }\end{array}$ \\
\hline Variables $^{\dagger}$ & $(n=2001)$ & $(n=1919)$ & $(n=3622)$ & $(n=779)$ \\
\hline \multicolumn{5}{|l|}{ Socio-demographic } \\
\hline Age, years, mean (SD) & $71.8(5.1)$ & $71.5(5.1)$ & $71.5(5.1)$ & $71.4(5.1)$ \\
\hline Education, > high school & $764(38.2)$ & $757(39.5)$ & $1429(39.5)$ & $222(28.5)$ \\
\hline Married & $965(48.2)$ & $963(50.2)$ & $1865(51.5)$ & $380(48.8)$ \\
\hline Living alone & $855(42.7)$ & $772(40.2)$ & $1445(39.9)$ & $331(42.5)$ \\
\hline \multicolumn{5}{|l|}{ Health-related } \\
\hline General health, excellent & $868(43.4)$ & $714(37.2)$ & $981(27.1)$ & $102(13.1)$ \\
\hline Current smoker & $157(7.9)$ & $141(7.4)$ & $279(7.7)$ & $81(10.4)$ \\
\hline Obese & $343(17.1)$ & $321(16.7)$ & $778(21.5)$ & $199(25.6)$ \\
\hline Prevalent vertebral fracture & $304(15.2)$ & $348(18.1)$ & $767(21.2)$ & $214(27.5)$ \\
\hline Arthritis & $892(44.6)$ & $1081(56.3)$ & $2591(71.5)$ & $666(85.5)$ \\
\hline Hip pain & $242(12.1)$ & $437(22.8)$ & $1365(37.7)$ & $515(66.1)$ \\
\hline Fall in last year & $465(23.2)$ & $546(28.5)$ & $1166(32.2)$ & $280(35.9)$ \\
\hline Hospitalized in last year & $230(11.5)$ & $264(13.8)$ & $555(15.3)$ & $186(23.9)$ \\
\hline Hypertension & $735(36.7)$ & $720(37.5)$ & $1344(37.1)$ & $344(44.2)$ \\
\hline Previous stroke & $41(2.1)$ & $52(2.7)$ & $113(3.1)$ & $41(5.3)$ \\
\hline Diabetes & $119(6.0)$ & $120(6.3)$ & $251(6.9)$ & $67(8.6)$ \\
\hline Breast cancer & $87(4.4)$ & $115(6.0)$ & $189(5.2)$ & $38(4.9)$ \\
\hline Breast surgery & $445(22.2)$ & $449(23.4)$ & $1034(28.6)$ & $208(26.7)$ \\
\hline Anxiety medication use & $149(7.4)$ & $207(10.8)$ & $577(15.9)$ & $182(23.4)$ \\
\hline Deaths after 16 years of follow-up & $1059(52.9)$ & $1043(54.4)$ & $1982(54.7)$ & $506(65.0)$ \\
\hline
\end{tabular}

Back pain exposure status defined by combining self-report of back pain presence and frequency at visit 1 (1986-1988) and visit 2 (1988-1990) + Data are presented as number (percentage) of patients unless otherwise indicated

Our findings were consistent with prior studies that found that older women with daily ${ }^{14}$ or disabling ${ }^{15}$ back pain had elevated mortality risk. Our finding that any/unspecified back pain reported at a single visit was not associated with mortality was also consistent with previous studies. ${ }^{8-12}$ Our findings corroborated studies showing other potentially disabling musculoskeletal conditions, such as hyperkyphosis ${ }^{26,27}$ and lower extremity osteoarthritis, ${ }^{28-30}$ are associated with elevated mortality risk.
This work extends research on disabling back pain and increased mortality. ${ }^{13,14}$ To our knowledge, our study is the first to measure disability after measurement of back pain, allowing prospective analysis of disability as a mediator of the association between back pain with mortality. Using causal mediation methods that incorporate interaction between exposure and mediator variables, ${ }^{24,} 25$ self-reported IADL impairment was an important mediator of this association. This finding agrees with studies of the associations of back pain

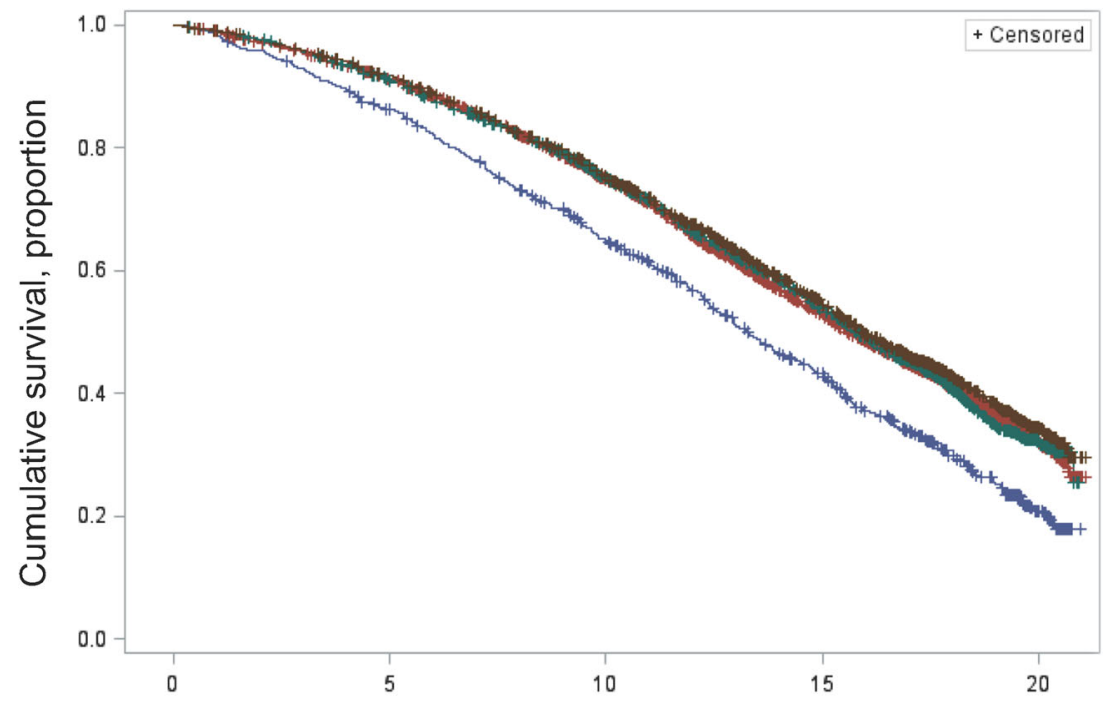

Time since visit 2 (1989-90), years

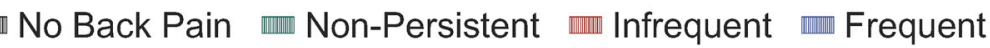

Fig. 2 Cumulative survival and back pain. 
Table 2 Hazard Ratios Between Back Pain and All-Cause and Cause-Specific Mortality Among 8321 Older Women in the Study of Osteoporotic Fractures, Visit 2 (1988-1990) to Visit 9 (2006-2008)

\begin{tabular}{|c|c|c|c|c|}
\hline All-cause mortality & $\begin{array}{l}\text { Death } \\
\text { rate* }\end{array}$ & $\begin{array}{l}\text { Age-adjusted } \\
\text { HR (95\% CI) }\end{array}$ & $\begin{array}{l}\text { Socio-demographic }{ }^{\dagger}- \\
\text { adjusted } \\
\text { HR }(95 \% \text { CI) }\end{array}$ & $\begin{array}{l}\text { Fully adjusted } \\
\text { HR }(95 \% \text { CI })\end{array}$ \\
\hline No back pain & 4280 & Ref & Ref & Ref \\
\hline Non-persistent back pain & 4439 & $1.05(0.96,1.14)$ & $1.05(0.97,1.14)$ & $1.01(0.93,1.10)$ \\
\hline Infrequent persistent back pain & 4487 & $1.04(0.97,1.12)$ & $1.04(0.97,1.12)$ & $0.98(0.91,1.05)$ \\
\hline Frequent persistent back pain & 5887 & $1.46(1.32,1.61)$ & $1.42(1.28,1.57)$ & $1.24(1.11,1.39)$ \\
\hline \multicolumn{5}{|l|}{ Cardiovascular mortality } \\
\hline No back pain & 1501 & Ref & Ref & Ref \\
\hline Non-persistent back pain & 1560 & $1.05(0.92,1.21)$ & $1.07(0.93,1.23)$ & $1.02(0.89,1.18)$ \\
\hline Infrequent persistent back pain & 1602 & $1.06(0.94,1.20)$ & $1.08(0.96,1.22)$ & $1.01(0.89,1.14)$ \\
\hline Frequent persistent back pain & 2299 & $1.62(1.37,1.91)$ & $1.57(1.33,1.85)$ & $1.34(1.12,1.61)$ \\
\hline \multicolumn{5}{|l|}{ Cancer mortality } \\
\hline No back pain & 798 & Ref & Ref & Ref \\
\hline Non-persistent back pain & 839 & $1.05(0.87,1.27)$ & $1.05(0.87,1.27)$ & $1.02(0.84,1.24)$ \\
\hline Infrequent persistent back pain & 865 & $1.08(0.92,1.27)$ & $1.08(0.92,1.27)$ & $1.03(0.87,1.23)$ \\
\hline Frequent persistent back pain & 1128 & $1.44(1.14,1.81)$ & $1.44(1.14,1.82)$ & $1.33(1.03,1.71)$ \\
\hline \multicolumn{5}{|l|}{ Pulmonary mortality } \\
\hline No back pain & 462 & Ref & Ref & Ref \\
\hline Non-persistent back pain & 495 & $1.08(0.85,1.39)$ & $1.10(0.86,1.40)$ & $1.02(0.80,1.31)$ \\
\hline Infrequent persistent back pain & 457 & $0.98(0.79,1.22)$ & $1.00(0.80,1.24)$ & $0.87(0.69,1.10)$ \\
\hline Frequent persistent back pain & 713 & $1.65(1.23,2.23)$ & $1.71(1.27,2.30)$ & $1.32(0.95,1.83)$ \\
\hline
\end{tabular}

Ref, reference; HR, hazard ratio; $C I$, confidence interval

*Deaths per 100,000 person years

†Sociodemographic-adjusted model: age, education, marital status, living alone, recruitment site

¥Fully adjusted model, sociodemographic + health-related characteristics: excellent general health, current smoker, obese, prevalent vertebral fracture, arthritis, hip pain, fall history, hospitalizations, hypertension, previous stroke, diabetes, history of breast cancer, previous breast surgery, and anxiety medication use

with disability in older adults, ${ }^{31-33}$ and disability (IADL impairments, poor mobility) with mortality in late-life. ${ }^{34-37}$ However, the mechanism of this association remains unclear. While back pain may directly impair IADLs, older adults may inappropriately avoid IADLs due to fear of re-injury or worsening of symptoms. Frequent back pain that persists over time and impairs IADLs may lead to weight gain, development or progression of comorbid conditions, and ultimately death. However, in each of our mediation analyses, we observed a residual direct effect of back pain on mortality, i.e., an effect not mediated by disability. Other mediators or persistent pain itself may affect mortality.

Our finding of moderately elevated cardiovascular mortality is consistent with two studies, one of older women ${ }^{14}$ and another of younger men. ${ }^{8}$ However, two additional studies of middle-aged men ${ }^{8}$ and older adults ${ }^{13}$ have found no increase in cardiovascular death. This is the first study, to our knowledge, to evaluate the association of back pain with cancer- or pulmonary-specific mortality in older women. It is possible that back pain was a symptom of bony metastasis. However, all participants in our sample were evaluated for current or previous cancer at baseline, including lumbar x-rays taken at the baseline visit, making this less likely. Older adults with back pain and IADL limitations may also limit physical activity, which is protective of both cancer and cardiovascular mortality. ${ }^{38,39}$ Similar higher rates of cancer-specific mortality have been observed in older adults with chronic pain ${ }^{40}$ and osteoarthritis. ${ }^{28}$ Degenerative changes of the spine, common in older adults, may lead to elevated levels of inflammatory cytokines, ${ }^{41,} 42,43$ which may contribute to cancer and cardiovascular death. ${ }^{41}$

Our study has several limitations. The sample was restricted to older, community-dwelling white women who were generally healthy, and may not be generalizable to younger adults, men, women of other races/ethnicities, or to older women already disabled or living in nursing homes. Additionally, participants were not followed from the onset of back pain, making it difficult to infer the causal pathway from back pain to mortality. Future research should prospectively examine the longitudinal impact of disability over time following the onset of back pain. We used a complete case analytic approach. While exclusion of participants missing baseline values of potential confounders may have biased our results, this seems unlikely as a low proportion of participants $(\sim 7 \%)$ were missing baseline data and baseline characteristics of excluded women were similar to those of our analytic sample. Residual confounding was possible for some variables, such as baseline osteoporosis. However, we adjusted for prevalent vertebral fractures, an important potential consequence of osteoporosis, at baseline using morphometry. Additionally, it seems unlikely that our estimates would change due to other baseline fragility fractures (e.g., hip or wrist), since we adjusted for fall history, a strong predictor of such fractures.

We did not adjust for baseline levels of pain medication use (e.g., NSAIDs, Opioids). Although pain medications may be associated with mortality, use of pain medications preceding back pain seems unlikely. A concern is potential confounding by pain medications used for other reasons; however, we 
Table 3 Mediation Analysis Between Frequent Persistent Back Pain at Visit 2 (1988-1990) and All-Cause Mortality at Visit 9 (2006-2008)

\begin{tabular}{|c|c|c|c|c|}
\hline$\overline{\text { Mediator* }}$ & $\begin{array}{l}\text { Total effect }^{\dagger} \\
(\mathrm{OR}, 95 \% \mathrm{CI})\end{array}$ & $\begin{array}{l}\text { Indirect effect }^{\dagger} \\
(\mathrm{OR}, 95 \% \mathrm{CI})\end{array}$ & $\begin{array}{l}\text { Direct effect }{ }^{\dagger} \\
(\text { OR, 95\% CI) }\end{array}$ & Proportion mediated, \% \\
\hline $\begin{array}{l}\text { IADL limitations }(n=7936) \\
\text { Performance-based physical function }\end{array}$ & $1.28(1.06,1.52)$ & $1.12(1.08,1.15)$ & $1.15(0.94,1.36)$ & $47 \%$ \\
\hline Slow chair stand speed $(n=6797)$ & $1.19(0.97,1.45)$ & $1.05(1.03,1.08)$ & $1.14(0.93,1.38)$ & $27 \%$ \\
\hline Slow walking speed $(n=6913)$ & $1.22(1.02,1.46)$ & $1.05(1.02,1.07)$ & $1.17(0.97,1.40)$ & $24 \%$ \\
\hline
\end{tabular}

IADL, instrumental activities of daily living; OR, odds ratio

*Information for mediator variables was obtained at visit 3 (1991)

$\uparrow$ Fully-adjusted model: age, education, marital status, living alone, recruitment site, excellent general health, current smoker, obese, prevalent vertebral fracture, arthritis, hip pain, fall history, hospitalizations, hypertension, previous stroke, diabetes, history of breast cancer, previous breast surgery, and anxiety medication use

partially addressed this by adjusting for other sources of musculoskeletal pain (i.e., hip pain, arthritis). It seems more likely that pain medication use would function as a mediator of mortality.

Psychosocial factors, particularly depression, are also concerns. While depression is a possible confounder, it is also a likely mediator. We were unable to assess depression in this study because $16 \%$ of SOF participants were missing data on the Geriatric Depression Scale (GDS), which was administered at visit 2 . However, a sensitivity analysis that included GDS score (high vs. low) and an indicator variable for missing GDS score showed a similar risk of all-cause mortality among women with frequent persistent back pain (HR 1.24, 95\% CI $1.10-1.38$ ) compared to no back pain. Instead of adjusting for GDS, we adjusted for self-rated health status which reflects psychological health among older adults. ${ }^{44,}{ }^{45}$ Future studies should explore depression and other possible mediators (e.g., psychosocial factors, physical activity, medication use, fragility fractures, inflammatory markers) and examine interactions between these factors and likely mediators (e.g., IADL impairments).

Strengths of our study include its large sample size, long follow-up period, and assessment of back pain frequency at two time points. Our results were consistent across multiple measures of back pain. Our analytic approach included causespecific mortality and contemporary mediation methods not previously used to assess the association of back pain and mortality. Survival and mediation methods controlled extensively for confounding, including variables not measured in previous studies (e.g., prevalent vertebral fractures).

The proportion of adults over the age of 65 is increasing rapidly in the USA and optimizing physical health and extending life for older adults is an important public health goal. Our findings raise the question of whether better management of back pain across the lifespan could prevent disability, improve quality of life, and ultimately extend life. These results lay the foundation for future longitudinal intervention studies to assess the long-term impact of back pain treatments and self-care strategies on these outcomes. For now, clinicians should assess physical function in older adults with back pain and recommend guideline-based management, which encourages use of less-invasive treatments. ${ }^{46}$ Important goals of back pain treatment in older adults include addressing IADL impairments and optimizing physical function.

In conclusion, we found frequent persistent back pain to be associated with all-cause, cardiovascular, and cancer mortality among older community-dwelling white women over a median follow-up period of 14.1 years. Much of the association was mediated by disability.

Acknowledgements: We thank the participants and staff of the Study of Osteoporotic Fractures and Tuhina Neogi MD PhD for her thoughtful review of this manuscript.

Corresponding Author: Eric J. Roseen, DC MSc; Department of Family Medicine Boston Medical Center, One Boston Medical Center Place, Boston, MA, USA (e-mail: Eric.Roseen@bmc.org).

Author Contributions Drs. Roseen and Fredman had full access to all of the data in the study and take responsibility for the integrity of the data and the accuracy of the data analysis.

Study concept and design: Roseen, LaValley, Saper, Felson, and Fredman.

Acquisition, analysis, or interpretation of data: Roseen, LaValley, Saper, Li, Felson, and Fredman.

Drafting of the manuscript: Roseen and Fredman.

Critical revision of the manuscript for important intellectual content: Roseen, LaValley, Saper, Li, Felson, and Fredman.

Statistical analysis: Roseen, LaValley, Li, and Fredman.

Obtained funding: $n / a$.

Administrative, technical, or material support: Roseen and Fredman. Study supervision: Roseen and Fredman.

Funding/Support The Study of Osteoporotic Fractures (SOF) is supported by National Institutes of Health funding. The National Institute on Aging (NIA) provides support under the following grant numbers: R01 AG005407, RO1 AR35582, RO1 AR35583, RO1 AR35584, RO1 AG005394, RO1 AG027574, RO1 AG027576, and R01 AG026720. Dr. Roseen is supported by a Ruth L. Kirschstein National Research Service Award (1F32AT009272) from the National Center for Complementary and Integrative Health (NCCIH) and by the Boston University Clinical and Translational Science Institute (CTSI) Clinical Research Training Program (1UL1TR001430).

\section{Compliance with Ethical Standards:}

This study was approved by the Institutional Review Boards at each SOF clinical site and at Boston University Medical Campus.

Conflict of Interest: The authors declare that they do not have a conflict of interest 
Disclaimer: The funding sources had no role in the design and conduct of the study; collection, management, analysis, and interpretation of the data; preparation, review, or approval of the manuscript; and decision to submit the manuscript for publication. The contents of this manuscript are solely the responsibility of the authors and do not necessarily represent the official views of NIA or NCCIH.

\section{REFERENCES}

1. GBD 2015 Disease and Injury Incidence and Prevalence Collaborators. Global, regional, and national incidence, prevalence, and years lived with disability for 310 diseases and injuries, 1990-2015: a systematic analysis for the Global Burden of Disease Study 2015. Lancet. 2016;388: 1545-1602.

2. Hoy D, Bain C, Williams G, et al. A systematic review of the global prevalence of low back pain. Arthritis Rheum 2012;64(6): 2028-2037.

3. Merrill SS, Seeman TE, Kasl SV, Berkman LF. Gender differences in the comparison of self-reported disability and performance measures. J Gerontol A Biol Sci Med Sci 1997;52(1):M19-26.

4. Fillingim RB, King CD, Ribeiro-Dasilva MC, Rahim-Williams B, Riley JL. Sex, gender, and pain: a review of recent clinical and experimental findings. J Pain. 2009; 10(5):447-485.

5. Makris UE, Fraenkel L, Han L, Leo-Summers L, Gill TM. Epidemiology of restricting back pain in community-living older persons. J Am Geriatr Soc 2011;59(4):610-614.

6. Patel KV, Guralnik JM, Dansie EJ, Turk DC. Prevalence and impact of pain among older adults in the United States: findings from the 2011 National Health and Aging Trends Study. Pain. 2013;154(12):2649-2657.

7. Di Iorio A, Abate M, Guralnik JM, et al. From chronic low back pain to disability, a multifactorial mediated pathway: the InCHIANTI study. Spine. 2007;32(26):E809-815.

8. Astrand NE, Isacsson So. Back pain, back abnormalities, and competing medical, psychological, and social factors as predictors of sick leave, early retirement, unemployment, labour turnover and mortality: A 22 year follow up of male employees in a Swedish pulp and paper company. Br J Ind Med. 1988; 45(6):387-395.

9. Penttinen J. Back pain and risk of fatal ischaemic heart disease: 13 year follow up of Finnish farmers. BMJ. 1994;309(6964): 1267-1268.

10. Heliovaara M, Makela M, Aromaa A, Impivaara O, Knekt P, Reunanen A. Low back pain and subsequent cardiovascular mortality. Spine. 1995;20(19):2109-2111.

11. Torrance N, Elliott AM, Lee AJ, Smith BH. Severe chronic pain is associated with increased 10 year mortality. A cohort record linkage study. Eur J Pain. 2010;14(4):380-386.

12. Kareholt I, Brattberg G. Pain and mortality risk among elderly persons in Sweden. Pain. 1998;77(3):271-278.

13. Fernandez M, Boyle E, Hartvigsen $\mathbf{J}$, et al. Is this back pain killing me? All-cause and cardiovascular-specific mortality in older Danish twins with spinal pain. Eur J Pain. 2017;21(5):938-948.

14. Zhu K, Devine A, Dick IM, Prince RL. Association of back pain frequency with mortality, coronary heart events, mobility, and quality of life in elderly women. Spine. 2007;32(18):2012-2018.

15. Docking RE, Fleming J, Brayne $\mathbf{C}$, et al. The relationship between back pain and mortality in older adults varies with disability and gender: results from the Cambridge City over-75s Cohort (CC75C) study. Eur J Pain. 2015; 19(4):466-472.

16. Jordan KP, Croft P. Mortality and cancer in patients with new musculoskeletal episodes: a cohort study. $\mathrm{Br} J$ Gen Pract. 2010;60(572):e105-111.

17. Jette AM. Toward a common language of disablement. J Gerontol A Biol Sci Med Sci 2009;64(11):1165-1168.

18. Cummings SR, Black DM, Nevitt MC, et al. Appendicular bone density and age predict hip fracture in women. The Study of Osteoporotic Fractures Research Group. JAMA. 1990;263(5):665-668.

19. Kado DM, Browner WS, Palermo L, Nevitt MC, Genant HK, Cummings SR. Vertebral fractures and mortality in older women: a prospective study. Study of Osteoporotic Fractures Research Group. Arch Intern Med. 1999; 159(11):1215-1220.

20. Ensrud KE, Nevitt MC, Yunis C, et al. Correlates of impaired function in older women. J Am Geriatr Soc. 1994; 42(5):481-489.

21. Cesari M, Kritchevsky SB, Penninx BW, et al. Prognostic value of usual gait speed in well-functioning older people - results from the Health,
Aging and Body Composition Study. J Am Geriatr Soc. 2005;53(10):1675-1680.

22. Cesari M, Kritchevsky SB, Newman AB, et al. Added value of physical performance measures in predicting adverse health-related events: results from the Health, Aging and Body Composition Study. J Am Geriatr Soc. 2009;57(2):251-259.

23. Allison PD. Survival Analysis Using SAS: A Practical Guide, Second Edition. Crary, NC. SAS Institute Inc. 2010.

24. Vanderweele TJ. Explanation in Causal Inference: Methods for Mediation and Interaction. New York, NY: Oxford University Press. 2015.

25. Valeri L, VanderWeele TJ. SAS macro for causal mediation analysis with survival data. Epidemiology. 2015;26(2):e23-24.

26. Kado DM, Huang MH, Karlamangla AS, Barrett-Connor E, Greendale GA. Hyperkyphotic posture predicts mortality in older communitydwelling men and women: a prospective study. J Am Geriatr Soc. 2004; 52(10): 1662-1667

27. Kado DM, Lui LY, Ensrud KE, et al. Hyperkyphosis predicts mortality independent of vertebral osteoporosis in older women. Ann Intern Med. 2009;150(10):681-687.

28. Nuesch E, Dieppe P, Reichenbach S, Williams S, Iff S, Juni P. All cause and disease specific mortality in patients with knee or hip osteoarthritis: population based cohort study. BMJ. 2011;342:d1165.

29. Barbour KE, Lui LY, Nevitt MC, et al. Hip osteoarthritis and the risk of all-cause and disease-specific mortality in older women: a populationbased cohort study. Arthritis Rheumatol. 2015;67(7): 1798-1805.

30. Hoeven TA, Leening MJ, Bindels PJ, et al. Disability and not osteoarthritis predicts cardiovascular disease: A prospective populationbased cohort study. Ann Rheum Dis. 2015; 74(4):752-756.

31. Weiner DK, Haggerty CL, Kritchevsky SB, et al. How does low back pain impact physical function in independent, well-functioning older adults? Evidence from the Health $\mathrm{ABC}$ Cohort and implications for the future. Pain Med. 2003;4(4):311-320.

32. Scudds RJ, Robertson JM. Empirical evidence of the association between the presence of musculoskeletal pain and physical disability in community-dwelling senior citizens. Pain. 1998;75(2-3):229-235.

33. Edmond SL, Felson DT. Function and back symptoms in older adults. J Am Geriatr Soc. 2003;51(12):1702-1709.

34. Cooper R, Strand BH, Hardy R, Patel KV, Kuh D. Physical capability in mid-life and survival over 13 years of follow-up: British birth cohort study. BMJ. 2014;348:g2219.

35. Feeny D, Huguet N, McFarland BH, Kaplan MS, Orpana H, Eckstrom E. Hearing, mobility, and pain predict mortality: a longitudinal population-based study. J Clin Epidemiol. 2012;65(7):764-777.

36. Ensrud KE, Lui LY, Paudel ML, et al. Effects of mobility and cognition on risk of mortality in women in late life: a prospective study. J Gerontol A Biol Sci Med Sci. 2016;71(6):759-765.

37. Studenski S, Perera S, Patel K, et al. Gait speed and survival in older adults. JAMA. 2011;305(1):50-58.

38. Arem H, Moore SC, Patel A, et al. Leisure time physical activity and mortality: a detailed pooled analysis of the dose-response relationship. JAMA Intern Med. 2015; 175(6):959-967.

39. Moore SC, Lee IM, Weiderpass E, et al. Association of Leisure-Time Physical Activity With Risk of 26 Types of Cancer in 1.44 Million Adults. JAMA Intern Med. 2016;176(6):816-825.

40. Smith D, Wilkie R, Uthman O, Jordan JL, McBeth J. Chronic pain and mortality: A systematic review. PLoS One. 2014;9(6):e99048.

41. Johnson ZI, Schoepflin ZR, Choi H, Shapiro IM, Risbud MV. Disc in flames: Roles of TNF- $\alpha$ and IL-1 $\beta$ in intervertebral disc degeneration. Eur Cells Mater. 2015;30:104-117.

42. Dudli S, Fields AJ, Samartzis D, Karppinen J, Lotz JC. Pathobiology of Modic changes. Eur Spine J. 2016;25(11):3723-3734.

43. Couzin-Frankel J. Inflammation bares a dark side. Science. 2010;330(6011):1621.

44. Pinquart M. Correlates of subjective health in older adults: a metaanalysis. Psychol Aging. 2001;16(3):414-426

45. Schnittker J. When Mental Health Becomes Health: Age and the Shifting Meaning of Self-Rated Health. Milbank Q. 2005; 83:397-423.

46. Qaseem A, Wilt TJ, McLean RM, Forciea MA; Clinical Guidelines Committee of the American College of Physicians. Noninvasive treatments for acute, subacute, and chronic low back pain: a clinical practice guideline from the American College of Physicians. Ann Intern Med. 2017; 166:514-530. 